\title{
Implementation of an Electronic Disease Surveillance System in Guinea, 2016-2018
}

\author{
Eileen Reynolds, Boubacar Diallo, Pia Macdonald
}

Social and Statistical Sciences, RTI International, Durham, North Carolina, United States

Objective

The objective is to share the progress and challenges in the implementation of the District Health Information Software Versi on 2 (DHIS 2) as an electronic disease surveillance system platform in Guinea, West Africa, to inform Global Health Security Agenda efforts to strengthen real-time surveillance in low- resource settings.

\section{Introduction}

The West Africa Ebola outbreak of 2014-2016 demonstrated the importance of strong disease surveillance systems and the severe consequences of weak capacity to detect and respond to cases quickly. Challenges in the transmission and management of surveillance data were one factor that contributed to the delay in detecting and confirming the Ebola outbreak [1]. To help address this challenge, we have collaborated with the U.S. Centers for Disease Control and Prevention (CDC), the Ministry of Health $(\mathrm{MOH})$ in Guinea, the World Health Organization and various partners to strengthen the disease surveillance system through the implementation of an electronic reporting system using an open source software tool, the District Health Information Software Version 2 (DHIS 2). These efforts are part of the Global Health Security Agenda objective to strengthen real-time surveillance [2]. This online system enables prefecture health offices to enter aggregate weekly disease reports from health facilities and for that information to be immediately accessible to designated staff at prefecture, regional and national levels.

Incorporating DHIS 2 includes several advantages for the surveillance system. For one, the data is available in real time and can be analyzed quickly using built-in data analysis tools within DHIS 2 or exported to other analysis tools. In contrast, the existing system of reporting using Excel spreadsheets requires the MOH to manually compile spreadsheets from all the 38 prefectures to have case counts for the national level.

For the individual case notification system, DHIS 2 enables a similar accessibility of information that does not exist with the current paper-based reporting system. Once a case notification form is completed in DHIS 2, the case-patient information is immediately accessible to the laboratories receiving specimens and conducting testing for case confirmation. The system is designed so that laboratories enter the date and time that a specimen is received, and any test results. The results are then immediately accessible to the reporting district and to the stakeholders involved including the National Health Security Agency and the Expanded Program on Vaccination. In addition, DHIS 2 can generate email and short message service (SMS) messages to notify concerned parties at critical junctures in the process, for example, when a laboratory result is available for a given case.

\section{Methods}

This presentation is based on review of project experience and documentation for a Global Health Security project in Guinea from 2015-2018. In addition, this includes a 2017 evaluation of the DHIS 2 pilot phase in two regions each having five prefectures.

\section{Results}

The use of DHIS 2 for aggregate and individual case reports for disease surveillance was piloted in two regions in Guinea in 2017 for a period of six months. An evaluation of the pilot phase indicated strong capacity at the Prefecture Level to use the sys tem for weekly aggregate disease reporting as evidenced by the high weekly reporting rates as well as an assessment of users' capacities. Challenges observed during the pilot phase included weak follow-up and ownership by the national level MOH, weak adherence by the laboratories to enter data on the receipt and test results of laboratory samples, and individual case reports not filed in all cases. In addition, the lack of uniformity of common data elements on the forms across different diseases made analysis and data quality more challenging. 
Following the evaluation of the pilot phase the MOH directed that the system should be used for aggregate weekly reporting, however that the individual case reporting in DHIS 2 should wait until improvements could be made in the case report forms. Prefectures have used DHIS 2 for weekly aggregate disease reporting starting in January 2018. In addition, the MOH plans to implement electronic individual case reporting in DHIS 2 starting in October 2018.

\section{Conclusions}

Progress to date includes nationwide use of DHIS 2 by all prefectures for the submission of weekly aggregate case reports. In addition, the new case report forms have been configured in DHIS 2 and a training of trainers has been conducted at the national level to begin the process of implementing the electronic case reporting nationwide.

Challenges include the continuation of parallel weekly disease reporting in Excel for an extended period after adoption of DHIS 2 resulting in lower timeliness of weekly reports in DHIS 2 in some prefectures, weak use of the system for data analysis, building capacity within the Ministry of Health to maintain the system without outside assistance, sufficient resources to pay for internet access and power back-up (such as solar power) to enable the health offices to effectively use the system, weak data privacy and security procedures, and the need to strengthen management of the national DHIS 2 server.

\section{Acknowledgement}

This work is supported by funding provided by the Centers for Disease Control and Prevention Cooperative Agreement 1U19GH001591-01. We acknowledge the support of the U.S. Centers for Disease Control and Prevention and the Ministry of Health of Guinea for their technical support and collaboration of in all aspects of the work. In addition, we thank all the health workers and partners that have contributed to the implementation of DHIS 2 in Guinea and to strengthening the disease surveillance system.

\section{References}

1. Ministère de la Santé-République de Guinée, Direction Nationale de la Prevention et Santé Communautaire, Division Prevention et Lutte Contre la Maladie. Plan de Renforcement de la Surveillance des Maladies à Potentiel Epidémique en Guinée (2015-2017), August 2015.

2. Global HSA. Real-Time Surveillance Action Package: GHSA Action Package Detect 2 \& 3. [cited 2018 Oct 3]. Available from: https://www.ghsagenda.org/packages/d2-3-real-time-surveillance 